\title{
Temperature Control System of Cold Storage
}

\author{
Ting $\mathrm{Lu}^{1}$ and Zeliang $\mathrm{Liu}^{2}$ \\ School of Electronic Engineering, Jiujiang University, Jiujiang, Jiangxi, China \\ 1'luting524@163.com, ²aptblaze@163.com
}

Keywords: IOT; Sensor; Temperature detection

Abstract. This design is a temperature detection and automatic control system based on STC89S52 micro controller for the cold storage. Mainly consists of a power supply, a temperature detection circuit, a system control circuit, actuator, the temperature display circuit, alarm circuit and other parts, using DS18B20 temperature sensor detects the current temperature of cold storage through the STC89S52 controller and auxiliary circuit to calculate the output, and the LCD display the temperature value; if the measurement value is upper or lower limit value, the system will alarm, and at the same time to start or stop the actuator, making the temperature quickly reached the preset range.

\section{Introduction}

Cold storage is using cooling facilities to create the proper humidity and temperature conditions of warehouse, also known as cold storage, processing and storage of agricultural and livestock products. It can get rid of the influence of climate, prolong the period of storage preservation of agricultural and livestock products, in order to regulate market supply. At present, if you want to speed up the refrigerator industry to safe, normative, environmental protection, energy saving, convenient operation, simple structure of the direction of development, we must to improve the automatic management level of the cold storage and management to be further strengthened, and the operation cost is greatly cut down. This paper design temperature control system is a simple operation, and is low-cost, easy to run. This system is DS18B20 digital sensor to detect temperature, STC89S52 microcontroller to control the cold storage temperature, with temperature, alarm and control temperature and other functions.

\section{Determination of the programme}

For cold storage temperature control system, and the following three schemes: 1 . Selection of FPGA programmable logic device, by using FPGA device can greatly shorten the design time of the system and make the products to market faster, and then to enhance the competition ability of the product. 2 PLC programmable logic device, the PLC as the control system, it is characterized by the processing of the signal time is short, the progress is fast, and timely. All the I/O input and output signal are selected photoelectric isolation, with good self identification function. 3 STC STC89S52 microcontroller, which is characterized by low power consumption, high performance, the price is cheaper, and and the traditional 8051 microcontroller are compatible. Using this design scheme, considering the practicality and price, the chips and devices are common components, the cheap price for the whole system construction, and the prospect of this system is good.

This design to STC89S52 SCM as the mastermind, DS18B20 temperature sensor range - 50 DEG to 120 DEG C, the measured temperature is sent to STC89S52 SCM, the MCU, the temperature in the display circuit is displayed in real time, the system recovery is 1602 LCD module display. This system can not only display the temperature can also be used to a specific temperature value is set out, to measure the temperature real-time monitoring and control, when the temperature is higher than the set temperature, it will immediately make corresponding temperature equipment work rapidly adjust the cold storage temperature, so as to achieve the constant temperature state, then the control system is stable operation, the control automation. 


\section{The Design of control system}

\section{Control system structure.}

The circuit of the control system is composed of the temperature detecting circuit, the executing control circuit, the displaying circuit, the alarming circuit and so on.. DS18B20 temperature detection, detection to the cold storage temperature will upload the data to the MCU, microcontroller will the detected temperature value and the temperature setting of the numerical comparison, if the set temperature value is higher than, the microcontroller will output a control signal to the relay drives the motor to run down, and yellow lantern light prompt; if the detected temperature is below the set range, the green light and motor does not work. At the same time, the microcontroller will detect the temperature values displayed on the LCD1602 display, so that people can see how the temperature is intuitive and the process of cooling control. Control system structure diagram shown in Figure 1.

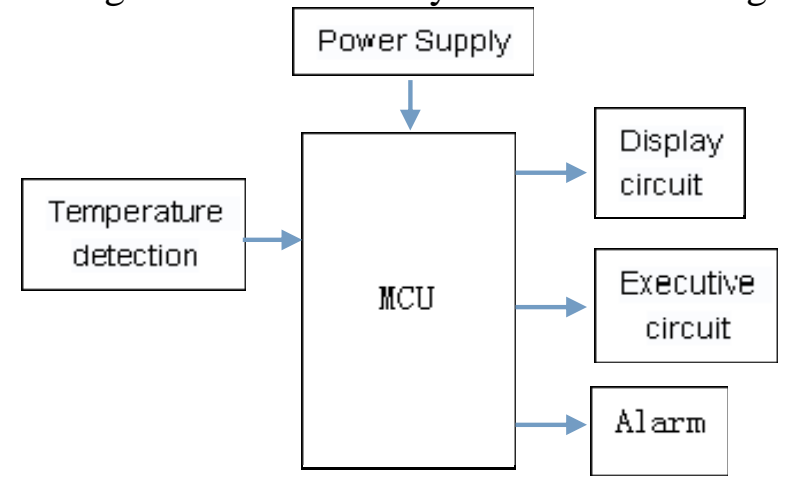

Fig.1. Structure of laboratory instruments management

\section{Temperature detection circuit}

The DS18B20 has four main data components: 1) 64-bit lasered ROM, 2) temperature sensor, 3) nonvolatile temperature alarm triggers $\mathrm{TH}$ and $\mathrm{TL}, 4)$ a configuration register. The device derives its power from the 1-Wire communication line by storing energy on an internal capacitor during periods of time when the signal line is high and continues to operate off this power source during the low times of the 1-Wire line until it returns high to replenish the parasite (capacitor) supply. As an alternative, the DS18B20 may also be powered from an external 3 volt - 5.5 volt supply. Communication to the DS18B20 is via a 1-Wire port. With the 1-Wire port, the memory and control functions will not be available before the ROM function protocol has been established.

The DS18B20 chip is used to realize the temperature detection. High integrated digital temperature sensor DS18B20 uses single bus type structure, has the advantages of small size, low power consumption and anti-interference. The DS18B20 Digital Thermometer provides 9 to 12-bit (configurable) temperature readings which indicate the temperature of the device. The core functionality of the DS18B20 is its direct-to-digital temperature sensor. The resolution of the DS18B20 is configurable $(9,10,11$, or 12 bits), with 12-bit readings the factory default state. This equates to a temperature resolution of $0.5^{\circ} \mathrm{C}, 0.25^{\circ} \mathrm{C}, 0.125^{\circ} \mathrm{C}$, or $0.0625^{\circ} \mathrm{C}$. A temperature conversion is performed and the thermal data is stored in the scratchpad memory in a 16-bit, sign-extended two's complement format. The temperature information can be retrieved over the 1-Wire interface by issuing a Read Scratchpad [BEh] command once the conversion has been performed. The data is transferred over the 1-Wire bus, LSB first. The MSB of the temperature register contains the "sign" (S) bit, denoting whether the temperature is positive or negative.

\section{Actuator}

SCM through the photoelectric switch and the transistor control relay on-off, the final control of the purpose of cooling. Figure 2 of the motor, when the temperature exceeds the limit indicated that the cooling device. When the temperature is lower than the set value, the microcontroller P3.7 sends a high level signal photoelectric switch no action, so the relay is disconnected, the motor does not rotate.

When the temperature exceeds the set temperature upper limit, SCM P3.7 send low level signal photoelectric switch action so that relay switch closed motor operated, achieve the purpose of cooling, 
when the temperature is lower than the set temperature value of the minimum MCU P3.7 sent a high level signal, electric machine stops running. Thus, the temperature can be stable in the setting temperature range.

The relay circuit has a protective circuit of the transistor, which is about to reverse the three ends of the tube.. Connect methods as shown in Figure 2. When the relay suddenly power off, the relay generates a large reverse current. The role of the diode is to reverse current diversion, so that the current flow through the three tube current is small, to protect the role of the transistor.

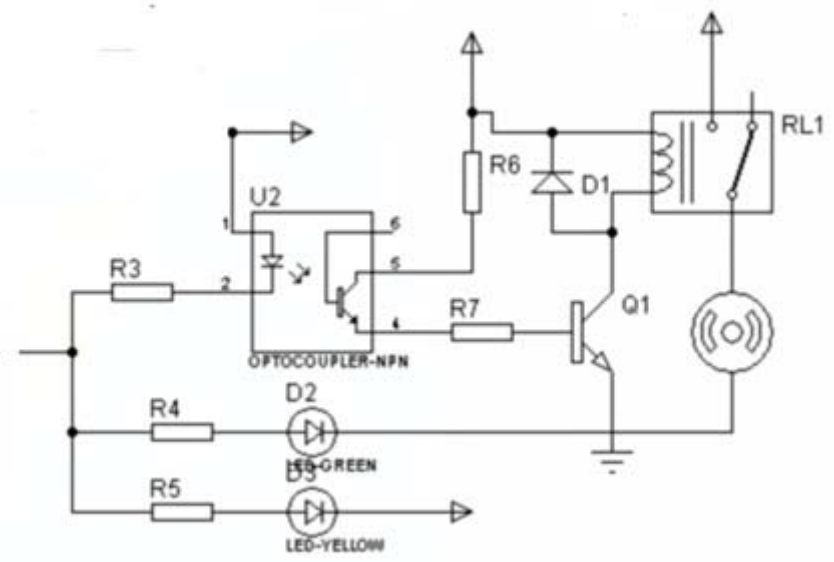

Fig.2. Actuator circuit

\section{Main program design flow chart}

The function of the program can be divided into two categories: one is the main program, it is the core of the whole control system, the purpose is to coordinate the relationship between the execution module and the operator. Two is a subroutine, which is used to complete a variety of substantive functions, such as measurement, calculation, display and other functions. The software subroutine module is not listed in this paper, and the flow chart of the main program is shown in Figure 3.

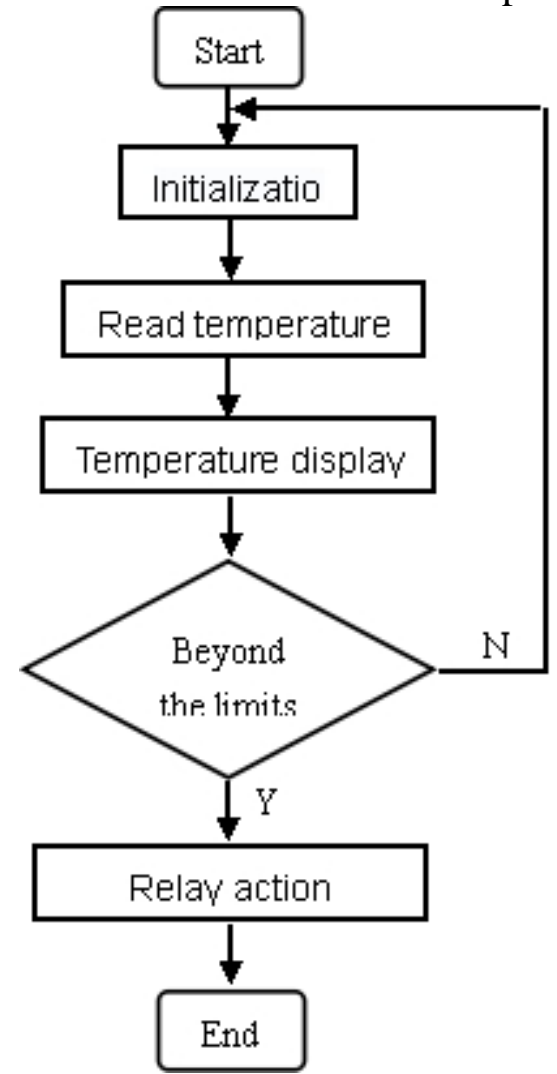

Fig.3. Flow chart of main program 


\section{Parameter function of display module LCD1602}

LCD1602 has 16 pin, but the main pin for program is only three: RS (data command select), R/W (read and write select), E (enable signal). RS is a register selection, high level select data register, low level select instruction register. R/W to read and write select, high level of reading, low level of write operation. E end to enable the end, the back and the timing associated with. In addition, the D0 D7 is 8 bit bidirectional data line.

Temperature values need to be shown to facilitate the monitoring and management of cold storage managers. This design uses LCD1602.P0 is the data port, P2 is the control port. Figure 4 is the flow chart of LCDl602.

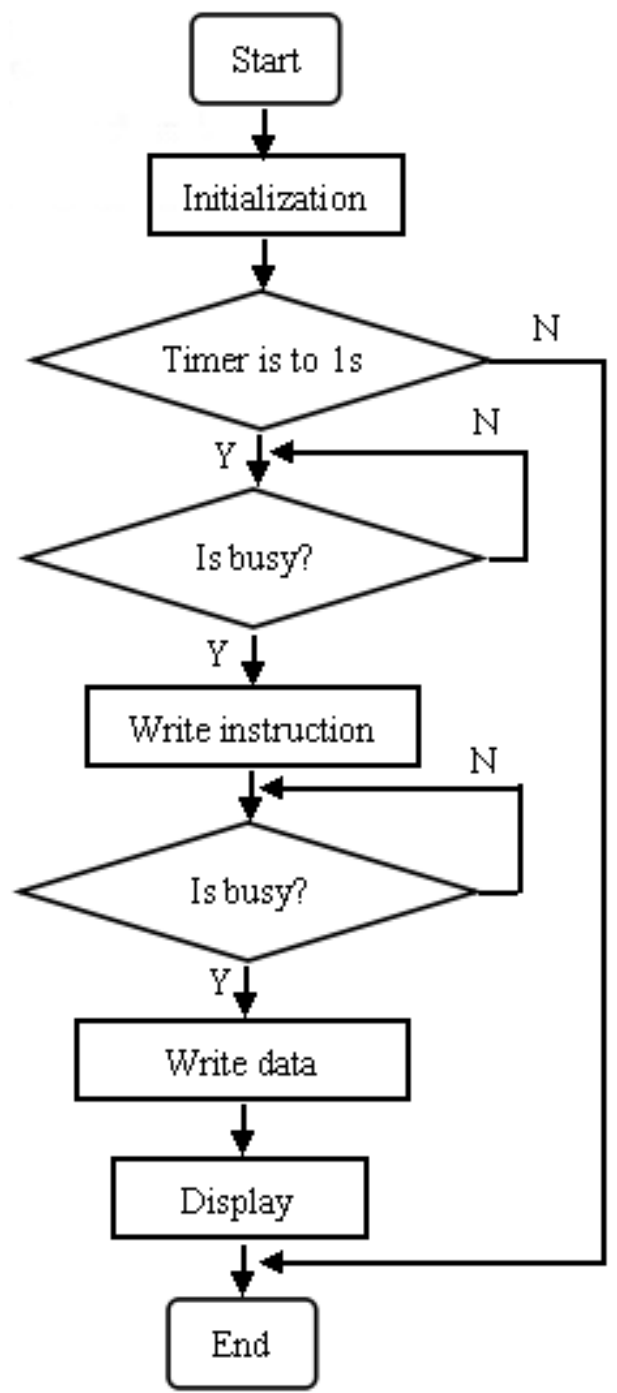

Fig.4. The flow chart of LCDl602

\section{Test results}

Frozen vegetables, fruits and dairy products, cold storage temperature is generally 5 to 13 degrees Celsius, when the temperature exceeds the set temperature upper limit, SCM P3.7 send low level signal photoelectric switch action so that relay switch closed motor operated, achieve the purpose of cooling, when temperature once again return to the set temperature value of the following microcontroller P3.7 sent a high level signal, the motor stopped running. As shown in Figure 5, when the temperature at 5 degrees Celsius, LCD1602 display is 16.5, the red light, the motor starts to rotate, the cold storage for cooling. The test shows that the design achieves the expected effect. 


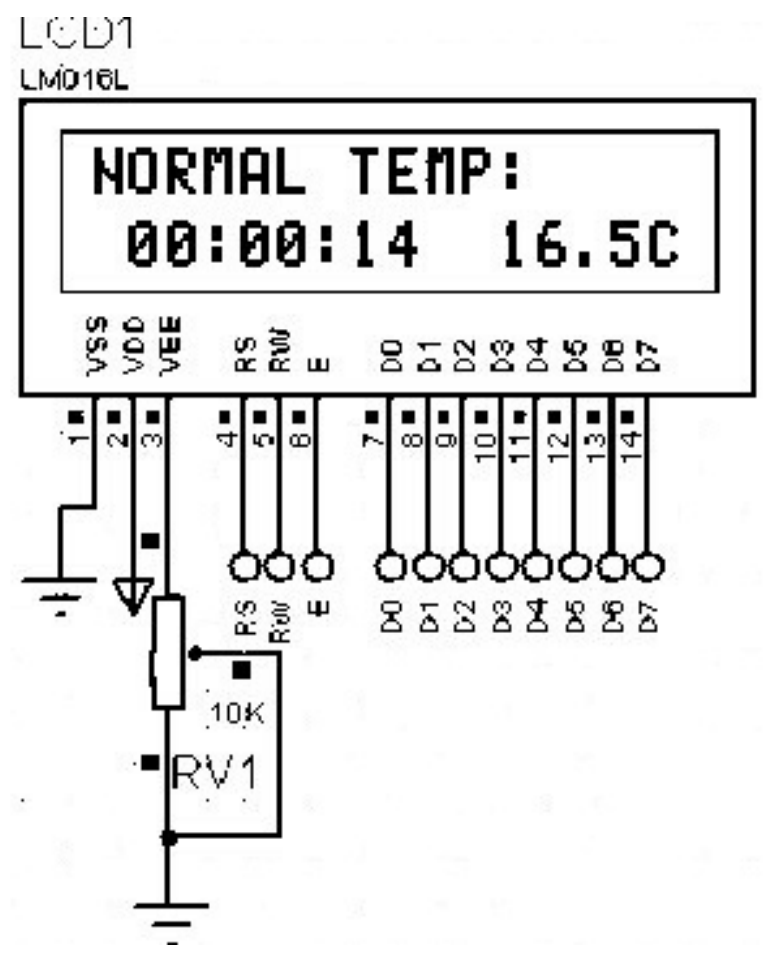

Fig.5. Test result

\section{Conclusion}

After the design of hardware circuit and the lower computer software, the system through the RS485 and PC (QT programming the interface) communication, the realization of the cold storage temperature monitoring system, managers can be shown by PC or LCD1602 LCD screen data real-time monitoring of grain temperature. This system can through PC set temperature warning value, also can display real-time temperature field sampling value, and save the results to the the database table of temperature information.

\section{Acknowledgements}

This work was financially supported by the Educational Commission of Jiangxi Province, China (GJJ14743), Scientific Research Fund of Jiujiang science and technology bureau (64) and Natural Science Foundation of Jiujiang University (2014KJYB002,2014KJYB006)..

\section{References}

[1] Chang Jiansheng. Detection and conversion technology [M]. Machinery Industry Press, 2011.

[2] Weng jiaming. Application development technology of MCU[M]. China power press, 2010.

[3] Wu Chunlei. Study on grain temperature and humidity intelligent detection system [D]. Tianjin: Hebei University of Technology, 2006.

[4] Fu Guicui, Gao Zexi. Temperature measurement design of high accuracy and multi-channel microcomputer control[J]. Measurement system, 2006.

[5] LU Yang. Temperature compensation approach of Pt resistance Temperature Measurement System[J]. Application Research of Computers, 2000.

[6] Yin Haikun. Research on the temperature control system based on single chip microcomputer [J]. technology to get rich guide, 2013, (11). 
[7] Ma Mingjian. Data acquisition and processing technology [M]. Xi'an: Xi'an Jiao Tong University press, 2012:1-2.

[8] Wu Jian, Hou Wen. The design of temperature control system [J]. scientific and technological information, 2012, (2): 35-38.

[9] Jing-Chiou Liou,Toward better software test estimates and requirement tracking, Journal of Computational Methods in Sciences and Engineering, 2011, 11,127 137.

[10] Jiang Shaochun, package Jun. Research and design of [J]. based on the temperature control system of vegetable greenhouse based on single chip microcomputer,2012,34 (9). 